\begin{tabular}{|c|l|}
\hline Title & A caution in the use of the NA FIC scoring system as a diagnostic screening tool for nonal coholic steatohepatitis \\
\hline Author(s) & $\begin{array}{l}\text { Nakamura, A kinobu; Y oneda, Masato; Sumida, Y oshio; Miyoshi, Hideaki; Nakajima, A tsushi; A tsumi, T atsuya; } \\
\text { Terauchi, Y asuo }\end{array}$ \\
\hline Citation & $\begin{array}{l}\text { Journal of Gastrointestinal \& Digestive System, 4(5), 221 } \\
\text { https://doi.org/L0.4172/2161-069X.1000221 }\end{array}$ \\
\hline Issue Date & 201410 \\
\hline Doc URL & http:/hdl.handle.net/2115/67263 \\
\hline Type & article \\
\hline File Information & JGastrointDigSyst4_221.pdf \\
\hline
\end{tabular}

Instructions for use 


\section{A Caution in the use of the NAFIC Scoring System as a Diagnostic Screening Tool for Nonalcoholic Steatohepatitis}

\author{
Akinobu Nakamura ${ }^{1}$, Masato Yoneda ${ }^{2}$, Yoshio Sumida ${ }^{3}$, Hideaki Miyoshi ${ }^{1}$, Atsushi Nakajima ${ }^{4}$, Tatsuya Atsumi ${ }^{1}$ and Yasuo Terauchi $^{\star}{ }^{*}$ \\ ${ }^{1}$ Division of Immunology and Metabolism, Hokkaido University Graduate School of Medicine, Sapporo, Japan \\ ${ }^{2}$ Schiff Center for Liver Diseases, University of Miami Miller School of Medicine, Miami, FL, USA \\ ${ }^{3}$ Department of Gastroenterology and Hepatology, Kyoto Prefectural University of Medicine, Kyoto, Japan \\ ${ }^{4}$ Department of Gastroenterology and Hepatology, Graduate School of Medicine, Yokohama City University, Yokohama, Japan \\ ${ }^{5}$ Department of Endocrinology and Metabolism, Graduate School of Medicine, Yokohama City University, Yokohama, Japan
}

*Corresponding author: Yasuo Terauchi, MD, PhD, Professor, Department of Endocrinology and Metabolism, Graduate School of Medicine, Yokohama City University, 3-9 Fukuura, Kanazawa-ku, Yokohama 236-0004, Japan, Tel: +81-45-787-2639; Fax: +81-45-784-3012; E-mail: terauchi-tky@umin.ac.jp

Received date: Aug 21, 2014, Accepted date: Sep 12, 2014, Published date: Sep 18, 2014

Copyright: (C) 2014 Nakamura A, et al. This is an open-access article distributed under the terms of the Creative Commons Attribution License, which permits unrestricted use, distribution, and reproduction in any medium, provided the original author and source are credited.

\begin{abstract}
Objective: We reinvestigated the clinical usefulness of the NAFIC and modified NAFIC scoring systems in Japanese patients with nonalcoholic fatty liver disease (NAFLD) according to the presence/absence of obesity.

Methods: A total of 141 Japanese patients with liver-biopsy-confirmed NAFLD were enrolled. All patients were classified as having nonalcoholic fatty liver (NAFL) or nonalcoholic steatohepatitis (NASH) on the basis of Matteoni's classification. Obesity was defined as a body mass index of $\geq 25$. To evaluate the overall accuracy of the NAFIC and modified NAFIC scoring systems, the sensitivity, specificity, positive predictive value (PPV) and negative predictive value (NPV) of these scoring systems for the diagnosis of NASH were calculated.

Results: In the obese group, the sensitivity, specificity, PPV and NPV of the NAFIC scoring system for the diagnosis of NASH were $67.3 \%, 76.2 \%, 77.8 \%$ and $65.3 \%$, respectively, while the corresponding values for the modified NAFIC scoring systems were $78.8 \%, 69.0 \%, 75.9 \%$ and $72.5 \%$. On the other hand, in the nonobese group, the sensitivity, specificity, PPV and NPV of the NAFIC scoring system were $47.1 \%, 86.7 \%, 66.7 \%$ and $74.3 \%$, respectively, while those of the modified NAFIC scoring system were $58.8 \%, 83.3 \%, 66.7 \%$ and $78.1 \%$, respectively. When the patients were divided by sex, the sensitivity of the NAFIC and modified NAFIC scoring systems in the female nonobese group were $53.8 \%$ and $69.2 \%$, respectively. However, surprisingly, in the male nonobese group, the sensitivity of both the scoring systems was only $25.0 \%$.
\end{abstract}

Conclusion: The sensitivity of both the NAFIC and modified NAFIC scoring systems for the diagnosis of NASH was lower in the male nonobese group than in all the other groups. These findings suggest that caution should be exercised in the use of the NAFIC scoring system as a diagnostic screening tool for NASH in Japanese patients with NAFLD, especially male nonobese patients.

Keywords: Body mass index; Female; Insulin; Male; Nonalcoholic fatty liver disease; Nonalcoholic steatohepatitis; Obesity; Sensitivity; Type IV collagen $7 \mathrm{~S}$

\section{Introduction}

Nonalcoholic fatty liver disease (NAFLD) is the most prevalent form of chronic liver disease in the world. It has been estimated that as many as $30 \%$ of adults in both Western countries and Japan have NAFLD [1,2]. The clinicopathologic spectrum of NAFLD extends from nonalcoholic fatty liver (NAFL) to nonalcoholic steatohepatitis (NASH). While NASH carries a high risk of liver disease-related mortality, such as death from hepatic cirrhosis and hepatocellular carcinoma [3,4], NAFL carries a low risk of liver disease-related mortality. Therefore, it is clinically important to distinguish NASH from NAFL.

While numerous non-invasive tests for differentiating NASH from NAFL have been developed [5], the NAFIC scoring system, based on the serum level of ferritin, fasting serum level of insulin and serum level of type IV collagen 7S, has been recognized as a simple and reasonably accurate and clinically useful tool for predicting the presence of NASH in Japanese patients with NAFLD [6]. Furthermore, a modified NAFIC scoring system has also been developed, modified by changing the weightage assigned to the fasting serum insulin level based on the importance of hyperinsulinemia in the pathogenesis of NASH [7-9]. We investigated the clinical usefulness of the modified NAFIC scoring system, and showed superior sensitivity of this modified scoring system for the diagnosis of NASH [10].

Existence of ethnic differences in the relationship between the body mass index (BMI) and liver fat content has been recognized. Azuma et al. showed that Japanese men had a greater predisposition for the development of a fatty liver even with a small increase of the BMI than non-Hispanic whites [11]. Also, according to health check findings in Japan, the prevalence of NAFLD is in the range of $10-20 \%$ in nonobese subjects $[2,12]$. This statistic suggests that differentiating NASH from NAFL could be important even in nonobese patients with NAFLD. 
Citation: Nakamura A, Yoneda M, Sumida Y, Miyoshi H, Nakajima A, et al. (2014) A Caution in the use of the NAFIC Scoring System as a

Page 2 of 7

In the present study, therefore, we reinvestigated the clinical usefulness of the NAFIC and modified NAFIC scoring systems in Japanese patients with NAFLD who had undergone liver biopsy stratified by the presence/absence of obesity.

\section{Methods}

\section{Patients}

A total of 141 patients with NAFLD confirmed by liver biopsy between 2004 and 2010 at Yokohama City University Hospital, Japan, were enrolled. The present study was conducted with the approval of the institutional review board, and written informed consent was obtained from all the patients participating in the study. A detailed history was obtained from every patient, and a careful physical examination was carried out. The histological criterion for the diagnosis of NAFLD is the presence of macrovesicular fatty change in the hepatocytes, with displacement of the nucleus to the edge of the cells [13]. The exclusion criteria were: a history of hepatic disease, including chronic hepatitis $\mathrm{C}$ or concurrent active hepatitis B (serum positive for hepatitis B surface antigen), autoimmune hepatitis, primary biliary cirrhosis, sclerosing cholangitis, hemochromatosis, $\alpha 1-$ antitrypsin deficiency or Wilson's disease, non-availability of data on difficulty in measuring the height and/or weight of the patient, and current/past consumption of more than $20 \mathrm{~g}$ of alcohol daily. None of the subjects enrolled in the study had a history of taking drugs that could cause fatty liver, such as amiodarone, diltiazem, tamoxifen or steroids. None of the subjects presented with clinical evidence of hepatic decompensation, such as hepatic encephalopathy, ascites, variceal bleeding or a serum bilirubin level more than twice the upper limit of normal.

\section{Clinical and laboratory evaluation}

Body weight and height were obtained for the subjects, and the BMI was calculated. Obesity was defined as a BMI of $\geq 25$ [14]. Venous blood samples were collected after the subjects had fasted overnight $(12 \mathrm{~h})$. Laboratory evaluations included measurements of the serum levels of aspartate aminotransferase (AST), alanine aminotransferase (ALT), ferritin, type IV collagen 7S, and insulin in all patients; all of these parameters were measured using standard techniques. The score in the original NAFIC scoring system is calculated as the weighted sum of three clinical variables [Serum ferritin $\geq 200 \mathrm{ng} / \mathrm{ml}$ (female) or $\geq 300 \mathrm{ng} / \mathrm{ml}$ (male), 1 point; serum fasting insulin $\geq 10 \mu \mathrm{U} / \mathrm{ml}, 1$ point; serum type IV collagen $7 \mathrm{~S} \geq 5.0 \mathrm{ng} / \mathrm{ml}, 2$ points] (Table $1 \mathrm{a}$ ). It has been demonstrated that the possibility of NASH is high when the NAFIC score is 2 or higher [6]. The modified NAFIC score is also a weighted sum of the scores for the three clinical variables, although the weightage given to higher fasting serum insulin levels is higher than that in the original NAFIC scoring system [Serum ferritin $\geq 200 \mathrm{ng} / \mathrm{ml}$ (female) or $\geq 300 \mathrm{ng} / \mathrm{ml}$ (male), 1 point; serum type IV collagen $7 \mathrm{~S} \geq$ $5.0 \mathrm{ng} / \mathrm{ml}, 2$ points; serum fasting insulin $10-15 \mu \mathrm{U} / \mathrm{ml}, 1$ point and $\geq$ $15 \mu \mathrm{U} / \mathrm{ml}, 2$ points] (Table $1 \mathrm{~b}$ ). It has been demonstrated that the possibility of NASH is high when the modified NAFIC score is 2 or higher [10].

\begin{tabular}{|l|l|l|}
\hline Clinical parameter & Definition & Point \\
\hline Fasting insulin $(\mu \mathrm{U} / \mathrm{ml})$ & $<10$ & 0 \\
\hline & $\geq 10$ & 1 \\
\hline Ferritin $(\mathrm{ng} / \mathrm{ml})$ & $<200$ (female) or <300 (male) & 0 \\
\hline & $\geq 200$ (female) or $\geq 300$ (male) & 1 \\
\hline Type IV collagen $7 \mathrm{~S}(\mathrm{ng} / \mathrm{ml})$ & $<5$ & 0 \\
\hline & $\geq 5$ & 2 \\
\hline
\end{tabular}

Table 1a: The criteria in the original NAFIC scoring system $[6,10]$.

\begin{tabular}{|l|l|l|}
\hline Clinical parameter & Definition & Point \\
\hline Fasting insulin $(\mu \mathrm{U} / \mathrm{ml})$ & $<10$ & 0 \\
\hline & 15 -Oct & 1 \\
\hline Ferritin $(\mathrm{ng} / \mathrm{ml})$ & $\geq 15$ & 2 \\
\hline & $<200$ (female) or <300 (male) & 0 \\
\hline Type IV collagen $7 \mathrm{~S}(\mathrm{ng} / \mathrm{ml})$ & $\geq 200$ (female) or $\geq 300$ (male) & 1 \\
\hline & $<5$ & 0 \\
\hline
\end{tabular}

Table 1b: The criteria in the modified NAFIC scoring system $[6,10]$. 
Citation: Nakamura A, Yoneda M, Sumida Y, Miyoshi H, Nakajima A, et al. (2014) A Caution in the use of the NAFIC Scoring System as a Diagnostic Screening Tool for Nonalcoholic Steatohepatitis. J Gastroint Dig Syst 4: 221. doi:10.4172/2161-069X.1000221

Page 3 of 7

\section{Histological evaluation}

All the patients enrolled in this study underwent a percutaneous liver biopsy under ultrasound guidance. The liver biopsy specimens were stained with hematoxylin-eosin, reticulin and Masson trichrome stains, and all the specimens were examined by an experienced pathologist who was blinded to the clinical and biochemical data of the patients. All the patients were classified as having steatosis or steatohepatitis on the basis of Matteoni's classification (type 1, simple steatosis without inflammation or fibrosis; type 2, steatosis with lobular inflammation but without fibrosis; type 3, additional presence of ballooned hepatocytes; type 4, presence of either Mallory's hyaline bodies or fibrosis) $[15,16]$. If the Matteoni classification was type 3 or 4 , the subject was diagnosed as having NASH.

\section{Statistical analysis}

The results are expressed as means \pm SD. The statistical significances of differences between two groups were analyzed by Student's $t$ test. The chi-square test was used to analyze the significance of differences in the proportions among groups. To evaluate the overall accuracy of the NAFIC score and the modified NAFIC score, the sensitivity, specificity, positive predictive value
(PPV) and negative predictive value (NPV) of the scoring systems for the diagnosis of NASH were calculated. A p value $<0.05$ was considered to indicate a statistically significant difference.

\section{Results}

\section{Characteristics of the patients}

The clinical and biochemical characteristics of the patients are shown in Table 2. Of the 141 patients, $94(66.7 \%)$ were diagnosed as having obesity (obese group), while the remaining 47 (33.3\%) were nonobese (nonobese group). There were no significant differences in the serum levels of AST or ferritin between the two groups. The age and the AST/ALT ratio were significantly lower in the obese group than in the nonobese group, whereas, by contrast, the percentage of males, the BMI, and the serum levels of AST, ALT, type IV collagen 7S and insulin were significantly higher in the obese group than in the nonobese group. Both the NAFIC scores and modified NAFIC scores were significantly higher in the obese group than in the nonobese group. Also, the percentage of patients with NASH was significantly higher in the obese group (55.3\%) than in the nonobese group (36.2\%).

\begin{tabular}{|l|l|l|l|}
\hline & Obese group & Nonobese group & p value \\
\hline $\mathrm{N}$ & 94 & 47 & \\
\hline Age (years) & $45.8 \pm 13.7$ & $55.4 \pm 14.4$ & 0.0002 \\
\hline Sex (male/female) & $62 / 32$ & $18 / 29$ & 0.0018 \\
\hline BMI $\left(\mathrm{kg} / \mathrm{m}^{2}\right)$ & $30.2 \pm 4.2$ & $22.8 \pm 1.5$ & $<0.0001$ \\
\hline AST $(\mathrm{IU} / \mathrm{l})$ & $50.0 \pm 24.7$ & $46.9 \pm 38.0$ & 0.6187 \\
\hline ALT $(\mathrm{IU} / \mathrm{l})$ & $87.1 \pm 53.9$ & $65.6 \pm 48.9$ & 0.0232 \\
\hline AST/ALT ratio & $0.63 \pm 0.17$ & $0.78 \pm 0.26$ & 0.0007 \\
\hline Ferritin $(\mathrm{ng} / \mathrm{ml})$ & $283.7 \pm 229.8$ & $232.7 \pm 232.8$ & 0.2183 \\
\hline Type IV collagen $7 \mathrm{~S}(\mathrm{ng} / \mathrm{ml})$ & $4.72 \pm 1.35$ & $4.13 \pm 0.84$ & 0.002 \\
\hline Fasting insulin $(\mu \mathrm{U} / \mathrm{ml})$ & $15.3 \pm 10.3$ & $10.4 \pm 5.0$ & 0.0002 \\
\hline NAFIC score & $1.69 \pm 1.33$ & $0.98 \pm 1.07$ & 0.0017 \\
\hline Modified NAFIC score & $2.03 \pm 1.59$ & $1.15 \pm 1.30$ & 0.0013 \\
\hline NASH/NAFL & $52 / 42$ & $17 / 30$ & 0.032 \\
\hline
\end{tabular}

Table 2: Clinical characteristics of all patients in the obese and nonobese groups; BMI: body mass index; AST: aspartate aminotransferase; ALT: alanine aminotransferase; NASH: nonalcoholic steatohepatitis; NAFL: nonalcoholic fatty liver; Values are expressed as means \pm S.D.

\section{Prediction of NASH from the NAFIC and modified NAFIC scores in the obese group}

The clinical and biochemical characteristics of the patients in the obese group are shown in Table 3. Of the 94 patients in this group, 52 (55.3\%) were histologically diagnosed as having NASH, and the remaining 42 (44.7\%) as not having NASH. The serum levels of AST, ALT, type IV collagen $7 \mathrm{~S}$ and insulin were significantly higher in the NASH group than in the NAFL group. Both the NAFIC score and modified NAFIC score were significantly higher in the NASH group than in the NAFL group. A $2 \times 2$ cross tabulation of the numbers of patients with and without NASH arranged in groups with NAFIC and modified NAFIC scores of $<2$ or $\geq 2$ is shown in Table 4 . The sensitivity, specificity, PPV and NPV of the NAFIC scoring system for the diagnosis of NASH were $67.3 \%, 76.2 \%, 77.8 \%$ and $65.3 \%$, respectively (Supplemental Table 1a); the corresponding values for the modified NAFIC scoring system were $78.8 \%, 69.0 \%, 75.9 \%$ and $72.5 \%$ (Supplemental Table 1b). These results showed that the sensitivity for the diagnosis of NASH increased with the use of the modified NAFIC score as compared to that of the NAFIC scoring system in the obese group, consistent with the finding in our previous study [10]. 
Citation: Nakamura A, Yoneda M, Sumida Y, Miyoshi H, Nakajima A, et al. (2014) A Caution in the use of the NAFIC Scoring System as a

\begin{tabular}{|l|l|l|l|}
\hline & NAFL & NASH & p value \\
\hline N & 42 & 52 & \\
\hline Age (years) & $43.2 \pm 12.3$ & $48.0 \pm 14.5$ & 0.0948 \\
\hline Sex (male/female) & $30 / 12$ & $32 / 20$ & 0.3144 \\
\hline BMI $\left(\mathrm{kg} / \mathrm{m}^{2}\right)$ & $29.5 \pm 4.0$ & $30.8 \pm 4.3$ & 0.1287 \\
\hline AST $($ IU/l) & $40.8 \pm 21.7$ & $57.4 \pm 24.8$ & 0.001 \\
\hline ALT $($ IU/l) & $73.2 \pm 48.0$ & $98.3 \pm 56.2$ & 0.0238 \\
\hline AST/ALT ratio & $0.61 \pm 0.15$ & $0.64 \pm 0.19$ & 0.3558 \\
\hline Ferritin $(\mathrm{ng} / \mathrm{ml})$ & $242.2 \pm 171.2$ & $317.2 \pm 264.9$ & 0.1012 \\
\hline Type IV collagen $7 \mathrm{~S}(\mathrm{ng} / \mathrm{ml})$ & $4.28 \pm 1.12$ & $5.07 \pm 1.43$ & 0.004 \\
\hline Fasting insulin $(\mu \mathrm{U} / \mathrm{ml})$ & $11.8 \pm 8.0$ & $18.1 \pm 11.1$ & 0.0022 \\
\hline NAFIC score & $1.02 \pm 1.09$ & $2.23 \pm 1.26$ & $<0.0001$ \\
\hline Modified NAFIC score & $1.19 \pm 1.27$ & $2.71 \pm 1.50$ & $<0.0001$ \\
\hline
\end{tabular}

Table 3: Clinical characteristics of the patients in the obese group

\begin{tabular}{|l|l|l|}
\hline NAFIC score & $\mathbf{0 - 1}$ & 4-Feb \\
\hline NAFL & 32 & 10 \\
\hline NASH & 17 & 35 \\
\hline
\end{tabular}

Table 4: The $2 \times 2$ cross tabulations of the numbers of patients with and without nonalcoholic steatohepatitis in the obese group arranged in groups with NAFIC scores of $<2$ or $\geq 2$ (a) and modified NAFIC scores of $<2$ or $\geq 2$

\begin{tabular}{|l|l|l|}
\hline Modified NAFIC score & $\mathbf{0 - 1}$ & $\mathbf{5 - F e b}$ \\
\hline NAFL & 29 & 13 \\
\hline NASH & 11 & 41 \\
\hline
\end{tabular}

Table 4: (b) NASH: nonalcoholic steatohepatitis; NAFL: nonalcoholic fatty liver

\section{Prediction of NASH from the NAFIC and modified NAFIC scoring systems in the nonobese group}

The clinical and biochemical characteristics of the patients in the nonobese group are shown in Table 5. Of the 47 patients in this group, 17 (36.2\%) were histologically diagnosed as having NASH, and the remaining $30(63.8 \%)$ as not having NASH. The serum levels of type IV collagen $7 \mathrm{~S}$ and insulin were significantly higher in the NASH group than in the NAFL group. Both the NAFIC scores and modified NAFIC scores were significantly higher in the NASH group than in the NAFL group. A $2 \times 2$ cross tabulation of the numbers of patients with and without NASH arranged in groups with NAFIC and modified
NAFIC scores of $<2$ or $\geq 2$ is shown in Table 6. The sensitivity, specificity, PPV and NPV of the NAFIC scoring system for the diagnosis of NASH were $47.1 \%, 86.7 \%, 66.7 \%$ and $74.3 \%$, respectively (Supplemental Table 2a); the corresponding values for the modified NAFIC scoring system were 58.8\%, 83.3\%, 66.7\% and $78.1 \%$, respectively (Supplemental Table $2 \mathrm{~b}$ ). These results indicate that the sensitivity of both the NAFIC and modified NAFIC scoring systems for the diagnosis of NASH was $20 \%$ lower in the nonobese group than that in the obese group, although the sensitivity increased with the use of the modified NAFIC score as compared to that of the NAFIC scoring system in the nonobese group.

\begin{tabular}{|l|l|l|l|}
\hline & NAFL & NASH & p value \\
\hline N & 30 & 17 & \\
\hline Age (years) & $52.5 \pm 14.7$ & $60.6 \pm 12.7$ & 0.0634 \\
\hline
\end{tabular}


Citation: Nakamura A, Yoneda M, Sumida Y, Miyoshi H, Nakajima A, et al. (2014) A Caution in the use of the NAFIC Scoring System as a Diagnostic Screening Tool for Nonalcoholic Steatohepatitis. J Gastroint Dig Syst 4: 221. doi:10.4172/2161-069X.1000221

Page 5 of 7

\begin{tabular}{|l|l|l|l|}
\hline Sex (male/female) & $14 / 16$ & $13-\mathrm{Apr}$ & 0.135 \\
\hline BMI $\left(\mathrm{kg} / \mathrm{m}^{2}\right)$ & $22.6 \pm 1.7$ & $23.1 \pm 1.1$ & 0.2313 \\
\hline AST $(\mathrm{IU} / \mathrm{l})$ & $40.3 \pm 34.5$ & $58.6 \pm 42.1$ & 0.1122 \\
\hline ALT $(\mathrm{IU} / \mathrm{l})$ & $55.5 \pm 42.3$ & $83.4 \pm 55.7$ & 0.0594 \\
\hline AST/ALT ratio & $0.78 \pm 0.24$ & $0.77 \pm 0.10$ & 0.8677 \\
\hline Ferritin $(\mathrm{ng} / \mathrm{ml})$ & $202.2 \pm 175.1$ & $286.9 \pm 308.9$ & 0.3087 \\
\hline Type IV collagen $7 \mathrm{~S}(\mathrm{ng} / \mathrm{ml})$ & $3.90 \pm 0.66$ & $4.55 \pm 0.97$ & 0.0085 \\
\hline Fasting insulin $(\mu \mathrm{U} / \mathrm{ml})$ & $9.0 \pm 3.7$ & $12.9 \pm 6.2$ & 0.0286 \\
\hline NAFIC score & $0.63 \pm 0.81$ & $1.59 \pm 1.23$ & 0.0084 \\
\hline Modified NAFIC score & $0.70 \pm 0.92$ & $1.94 \pm 1.52$ & 0.0055 \\
\hline
\end{tabular}

Table 5: Clinical characteristics of the patients in the nonobese group BMI: body mass index; AST: aspartate aminotransferase; ALT: alanine aminotransferase; NASH: nonalcoholic steatohepatitis; NAFL: nonalcoholic fatty liver; Values are expressed as means \pm S.D.

\begin{tabular}{|l|l|l|}
\hline NAFIC score & $\mathbf{0 - 1}$ & 4-Feb \\
\hline NAFL & 26 & 4 \\
\hline NASH & 9 & 8 \\
\hline
\end{tabular}

Table 6: The $2 \times 2$ cross tabulations of the numbers of patients with and without nonalcoholic steatohepatitis in the nonobese group arranged in groups with NAFIC scores of $<2$ or $\geq 2$ (a) and modified NAFIC scores of $<2$ or $\geq 2$

\begin{tabular}{|l|l|l|}
\hline Modified NAFIC score & $\mathbf{0 - 1}$ & $\mathbf{5}$-Feb \\
\hline NAFL & 25 & 5 \\
\hline NASH & 7 & 10 \\
\hline
\end{tabular}

Table 6: (b) NASH: nonalcoholic steatohepatitis; NAFL: nonalcoholic fatty liver

When the patients were divided by sex, the sensitivity of the NAFIC and modified NAFIC scoring systems in the female nonobese group were $53.8 \%$ and $69.2 \%$, respectively. However, surprisingly, in the male nonobese group, the sensitivity of both the scoring systems was only $25.0 \%$. Whereas, the sensitivity of the NAFIC and modified NAFIC scoring systems were $60.0 \%$ and $81.3 \%$ in the female obese group, and $71.9 \%$ and $75.0 \%$ in the male obese group, respectively. The clinical parameters of the NAFIC and modified NAFIC scoring systems stratified by the sex in the nonobese group are shown in Table 7. Although the serum levels of type IV collagen $7 \mathrm{~S}$ and fasting serum insulin levels, and the NAFIC and modified NAFIC scores were significantly higher in the female patients with NASH than in the female patients without NASH, there were no significant differences in these parameters or scores between the male patients with and without NASH. However, the NAFIC and modified NAFIC scores were significantly higher in the male obese patients with NASH than in those without NASH (NAFIC score: $0.97 \pm 0.89$ without NASH vs. $1.88 \pm 0.87$ with NASH, $\mathrm{P}=0.0001$; Modified NAFIC score: $1.17 \pm$ 1.15 without $\mathrm{NASH}$ vs. $2.38 \pm 1.13$ with $\mathrm{NASH}, \mathrm{P}=0.0001)$.

\begin{tabular}{|l|l|l|l|}
\hline & NAFL & NASH & p value \\
\hline Ferritin $(\mathrm{ng} / \mathrm{ml})$ & $270.2 \pm 228.6$ & $290.0 \pm 176.7$ & 0.8758 \\
\hline Type IV collagen 7S $(\mathrm{ng} / \mathrm{ml})$ & $3.97 \pm 0.78$ & $3.98 \pm 0.62$ & 0.9934 \\
\hline Fasting insulin $(\square \mathrm{U} / \mathrm{ml})$ & $9.7 \pm 3.8$ & $8.1 \pm 3.4$ & 0.4393 \\
\hline NAFIC score & $0.93 \pm 1.00$ & $0.75 \pm 0.96$ & 0.7545 \\
\hline
\end{tabular}


Citation: Nakamura A, Yoneda M, Sumida Y, Miyoshi H, Nakajima A, et al. (2014) A Caution in the use of the NAFIC Scoring System as a Diagnostic Screening Tool for Nonalcoholic Steatohepatitis. J Gastroint Dig Syst 4: 221. doi:10.4172/2161-069X.1000221

Page 6 of 7

\begin{tabular}{|l|l|l|l|}
\hline Modified NAFIC score & $1.00 \pm 1.11$ & $0.75 \pm 0.96$ & 0.6892 \\
\hline
\end{tabular}

Table 7: The clinical parameters of the NAFIC and modified NAFIC scoring systems stratified into males (a) and females

\begin{tabular}{|l|l|l|l|}
\hline & NAFL & NASH & p value \\
\hline Ferritin $(\mathrm{ng} / \mathrm{ml})$ & $142.3 \pm 76.6$ & $285.9 \pm 345.5$ & 0.1655 \\
\hline Type IV collagen 7S $(\mathrm{ng} / \mathrm{ml})$ & $3.83 \pm 0.55$ & $4.73 \pm 1.01$ & 0.01 \\
\hline Fasting insulin $(\square \mathrm{U} / \mathrm{ml})$ & $8.4 \pm 3.7$ & $14.4 \pm 6.1$ & 0.0033 \\
\hline NAFIC score & $0.38 \pm 0.50$ & $1.85 \pm 1.21$ & 0.0009 \\
\hline Modified NAFIC score & $0.44 \pm 0.63$ & $2.31 \pm 1.49$ & 0.0007 \\
\hline
\end{tabular}

Table 7: (b) in the nonobese group; NASH: nonalcoholic steatohepatitis; NAFL: nonalcoholic fatty liver

\section{Discussion}

In the present study, we estimated the clinical usefulness of the NAFIC and modified NAFIC scoring systems for distinguishing between NASH and NAFL according to the presence or absence of obesity in Japanese patients with nonalcoholic fatty liver disease (NAFLD). The results revealed that the sensitivity of both the NAFIC and modified NAFIC scoring systems for the diagnosis of NASH in obese Japanese patients with NAFLD was higher than or comparable to that in our previous study [10]. Therefore, these scoring systems, especially modified NAFIC scoring system, could be a clinically useful diagnostic screening tool for NASH in obese Japanese patients with NAFLD.

Whereas, our results also revealed that the sensitivity of both the NAFIC and modified NAFIC scoring systems for the diagnosis of NASH was low in nonobese Japanese patients with NAFLD. Although the prevalence of NAFLD by ultrasonography increased with the BMI in Japan according to an analysis of findings in health check findings [17], 10-20\% of Japanese nonobese subjects are estimated to have NAFLD $[2,12]$. Ethnic differences in the prevalence of hepatic steatosis have been noted in an urban population in the United States [18]. To investigate an ethnic differences in the relationship between BMI and liver fat content, Azuma et al. compared the liver fat content, estimated by computed tomography, in 313 Japanese men and 288 non-Hispanic whites [11]. They showed that liver fat content was higher in Japanese than in non-Hispanic white men, despite the lower mean BMI of Japanese men, and that the liver fat content increased at a higher rate with increasing BMI in Japanese men. Therefore, it is important to point out the problem of the low sensitivity of the NAFIC and modified NAFIC scoring systems in nonobese Japanese patients with NAFLD. In our study, $36.2 \%$ of nonobese patients with NAFLD were diagnosed as having NASH. Also, several studies have shown that BMI is not a useful independent factor for differentiating NAFL from NASH in Japanese patients with NAFLD $[6,19,20]$. Based on these perspectives, an accurate tool for differentiating NAFL from NASH would be needed even in nonobese patients with NAFLD.

Why was the sensitivity as estimated by the NAFIC and modified NAFIC scoring systems low in male nonobese Japanese patients with NAFLD? In our study, both the NAFIC and modified NAFIC scores were significantly higher in female nonobese patients with NASH than in those without NASH, while this was not the case in the male nonobese patients (Table 7). The NAFIC and modified NAFIC scoring systems are composed of the serum level of ferritin, fasting serum level of insulin and serum level of type IV collagen 7S. As shown in Table 7, the serum levels of type IV collagen $7 \mathrm{~S}$ and insulin were significantly higher in patients with NASH than in those without NASH in the female nonobese group, but not in the male nonobese group (Table 7). These results suggest that the absence of increases in the serum levels of type IV collagen $7 \mathrm{~S}$ or insulin in the male nonobese Japanese patients with NASH could be the reason for the low NAFIC and modified NAFIC scores in the male nonobese patients, resulting in the decreased sensitivity of either scoring system for the diagnosis of NASH. Currently, it is not known why there were differences in these parameters between the two sexes. However, since in clinical practice, it is important not to miss the presence of NASH, other noninvasive assessment tools, such as transient elastography [21], could be used for predicting NASH in male nonobese Japanese patients with NAFLD.

The limitations of our study were its retrospective design and the relatively small size of the sample. Another was that the patients were recruited from a hepatology center in Japan with a particular interest in the study of NAFLD, which could have introduced some referral bias. Patient selection bias could also have existed, because liver biopsy may have been considered only for NAFLD patients who were likely to have NASH. Thus, the findings may not entirely represent all NAFLD patients in the general population. In addition, it is not clear what the determinative factors are for distinguishing between NASH and NAFL in male nonobese Japanese patients with NAFLD. Therefore, one of the challenges for the future is to explore these factors. Another is to develop a simple noninvasive scoring system aimed at distinguishing between NASH and NAFL in male nonobese Japanese patients with NAFLD.

In conclusion, the sensitivities of both the NAFIC and modified NAFIC scoring systems for the diagnosis of NASH were lower in the male nonobese group than in the other groups. These findings suggest that caution should be exercised in the use of the NAFIC scoring system as a diagnostic screening tool for NASH in Japanese patients with NAFLD, especially male nonobese patients.

\section{Acknowledgments}

This work was supported in part by Grants-in-Aid for Scientific Research (B) 21390282 and (B) 24390235 from the Ministry of Education, Culture, Sports, Science and Technology (MEXT), of Japan, a Medical Award from the Japan Medical Association, a Grant- 
Citation: Nakamura A, Yoneda M, Sumida Y, Miyoshi H, Nakajima A, et al. (2014) A Caution in the use of the NAFIC Scoring System as a Diagnostic Screening Tool for Nonalcoholic Steatohepatitis. J Gastroint Dig Syst 4: 221. doi:10.4172/2161-069X.1000221

Page 7 of 7

in-Aid from the Uehara Memorial Foundation, a Grant-in-Aid from the Takeda Science Foundation (to Y.T.), and a Grant-in-aid for Front Runner of Future Diabetes Research (to A.N.). The authors have no conflicts of interests to declare.

\section{References}

1. Chalasani N, Younossi Z, Lavine JE, Diehl AM, Brunt EM, et al. (2012) The diagnosis and management of non-alcoholic fatty liver disease: Practice guideline by the American Gastroenterological Association, American Association for the Study of Liver Diseases, and American College of Gastroenterology. Gastroenterology 142: 1592-1609.

2. Eguchi Y, Hyogo H, Ono M, Mizuta T, Ono N, et al. (2012) Prevalence and associated metabolic factors of nonalcoholic fatty liver disease in the general population from 2009 to 2010 in Japan: a multicenter large retrospective study. J Gastroenterol 47: 586-595.

3. Starley BQ, Calcagno CJ, Harrison SA (2010) Nonalcoholic fatty liver disease and hepatocellular carcinoma: a weighty connection. Hepatology 51: $1820-1832$.

4. Ratziu V, Bellentani S, Cortez-Pinto H, Day C, Marchesini G (2010) A position statement on NAFLD/NASH based on the EASL 2009 special conference. J Hepatol 53: 372-384.

5. Yilmaz Y, Ulukaya E (2011) Toward a biochemical diagnosis of NASH: insights from pathophysiology for distinguishing simple steatosis from steatohepatitis. Curr Med Chem 18: 725-732.

6. Sumida Y, Yoneda M, Hyogo H, Yamaguchi K, Ono M, et al. (2011) A simple clinical scoring system using ferritin, fasting insulin, and type IV collagen $7 \mathrm{~S}$ for predicting steatohepatitis in nonalcoholic fatty liver disease. J Gastroenterol 46: 257-268.

7. Nakamura A, Yoneda M, Fujita K, Tajima K, Kikuchi K, et al. (2011) Impact of glucose tolerance on the severity of non-alcoholic steatohepatitis. J Diabetes Investig 2: 483-489.

8. Nakamura A, Tajima K, Zolzaya K, Sato K, Inoue R, et al. (2012) Protection from non-alcoholic steatohepatitis and liver tumourigenesis in high fat-fed insulin receptor substrate-1-knockout mice despite insulin resistance. Diabetologia 55: 3382-3391.

9. Nakamura A, Terauchi Y (2013) Lessons from mouse models of high-fat diet-induced NAFLD. Int J Mol Sci 14: 21240-21257.

10. Nakamura A, Yoneda M, Sumida Y, Eguchi Y, Fujii H, et al. (2013) Modification of a simple clinical scoring system as a diagnostic screening tool for non-alcoholic steatohepatitis in Japanese patients with nonalcoholic fatty liver disease. J Diabetes Investig 4: 651-658.

11. Azuma K, Kadowaki T, Cetinel C, Kadota A, El-Saed A, et al. (2009) Higher liver fat content among Japanese in Japan compared with nonHispanic whites in the United States. Metabolism 58: 1200-1207.

12. Amarapurkar DN, Hashimoto E, Lesmana LA, Sollano JD, Chen PJ, et al. (2007) How common is non-alcoholic fatty liver disease in the AsiaPacific region and are there local differences? J Gastroenterol Hepatol 22: 788-793.

13. Sanyal AJ American Gastroenterological Association (2002) AGA technical review on nonalcoholic fatty liver disease. Gastroenterology 123: 1705-1725.

14. Examination Committee of Criteria for 'Obesity Disease' in Japan; Japan Society for the Study of Obesity (2002) New criteria for 'obesity disease' in Japan. Circ J 66: 987-992.

15. Matteoni CA, Younossi ZM, Gramlich T, Boparai N, Liu YC, et al. (1999) Nonalcoholic fatty liver disease: a spectrum of clinical and pathological severity. Gastroenterology 116: 1413-1419.

16. Neuschwander-Tetri BA, Caldwell SH (2003) Nonalcoholic steatohepatitis: summary of an AASLD Single Topic Conference. Hepatology 37: 1202-1219.

17. Hashimoto E, Tokushige K (2011) Prevalence, gender, ethnic variations, and prognosis of NASH. J Gastroenterol 46 Suppl 1: 63-69.

18. Browning JD, Szczepaniak LS, Dobbins R, Nuremberg P, Horton JD, et al. (2004) Prevalence of hepatic steatosis in an urban population in the United States: impact of ethnicity. Hepatology 40: 1387-1395.

19. Shimada M, Kawahara H, Ozaki K, Fukura M, Yano H, et al. (2007) Usefulness of a combined evaluation of the serum adiponectin level, HOMA-IR, and serum type IV collagen 7S level to predict the early stage of nonalcoholic steatohepatitis. Am J Gastroenterol 102: 1931-1938.

20. Sumida Y, Yoneda M, Hyogo H, Itoh Y, Ono M, et al. (2012) Validation of the FIB4 index in a Japanese nonalcoholic fatty liver disease population. BMC Gastroenterol 12: 2.

21. Yoneda M, Yoneda M, Mawatari H, Fujita K, Endo H, et al. (2008) Noninvasive assessment of liver fibrosis by measurement of stiffness in patients with nonalcoholic fatty liver disease (NAFLD). Dig Liver Dis 40: 371-378. 